\title{
Deformed Quantum Field Theory, Thermodynamics at Low and High Energies, and Gravity. II. Deformation Parameter
}

\author{
A.E. Shalyt-Margolin \\ National Centre of Particles and High Enerogy Physics, Bogdanovich Str. 153, Minsk, 220040, Belarus
}

\begin{abstract}
The principal objective of this paper is to show that deformation of the General Relativity within the scope of both the Generalized Uncertainty Principle (UV-cutoff) and the Extended Uncertainty Principle (IR-cutoff) in the cases when the corresponding gravitational equations have a thermodynamic interpretation may be studied in terms of a small parameter introduced in previous works of the author. From this viewpoint the parameter is though to be universal. Consideration is given to the possibility for extension of the obtained results to more general cases. Possible generalization of the uncertainty relation for the pair (cosmological constant, "space-time volume"), where the cosmological constant is regarded as a dynamic quantity at high and low energies, is analyzed. This paper is devoted to the 70-th Anniversary of Professor Nikolai Maksimovich Shumeiko, Director of the Belarusian Particles and High Energy Physics National Centre.
\end{abstract}

Keywords Quantum Field Theory with UV and IR Cutoff, Gravitational Thermodynamics, Deformed Gravity

\section{Introduction}

In the last decade numerous works devoted to a Quantum Field Theory (QFT) at Planck's scale[1-3] have been published (of course, the author has no pretensions of being exhaustive in his references). This interest stems from the facts that (i) at these scales it is expected to reveal the effects of a Quantum Gravity (QG), and this still unresolved theory is intriguing all the researchers engaged in the field of theoretical physics; (ii) modern accelerators, in particular LHC, have the capacity of achieving the energies at which some QG effects may be exhibited.

Now it is clear that Quantum Field Theory (QFT) at Planck's scales, and possibly at very large scales as well, undergoes changes associated with the appearance of additional parameters related to (i) a minimal length (on the order of the Planck's length) and (ii)a minimum momentum. As this takes place, the corresponding parameters are naturally considered as deformation parameters, i.e. the related quantum theories are considered as a high-energy deformation (at Planck's scales) and a low-energy deformation (IR-cutoff), respectively, of the well-known quantum field theory, the latter being introduced in the corresponding highand low-energy limits and exact to a high level. The deformation is understood as an extension of a particular theory by

* Corresponding author:

a.shalyt@mail.ru (A.E. Shalyt-Margolin)

Published online at http://journal.sapub.org/ijtmp

Copyright (C) 2012 Scientific \& Academic Publishing. All Rights Reserved inclusion of one or several additional parameters in such a way that the initial theory appears in the limiting transition [4].

Most natural approach to the introduction of the abovementioned parameters is to treat a quantum field theory with the Generalized Uncertainty Principle (GUP) [5-15] and with the Extended Uncertainty Principle (EUP), respectively [1215]. In the case of GUP we easily obtain a minimal length on the order of the Planck's $l_{\min } \sim l_{p}$ and the corresponding high-energy deformation of well-known QFT-QFT with GUP. It should be noted that QFT with GUP at Planck's scales (Early Universe) is attested in many works (for example[5-11])). Even if we disregard the works devoted to a string theory, still remaining a tentative one, GUP is quite naturally derived from the gedanken experiment[6-9].

On the other hand, GUP has no way in the spaces with large length scales (for example (A)dS). For such spaces, e.g., in $[12,14]$ the Extended Uncertainty Principle has been introduced (find its exact definition below) giving an absolute minimum in the uncertainty of the momentum.

The problem is to find whether there are cases when the deformations generated by GUP and EUP are defined by the same parameter. By author's opinion this is the case for Gravity modified (deformed) within GUP and EUP, when the corresponding initial theory has a "thermodynamic interpretation"[16-21]. Specifically, the deformation parameter $\alpha=l_{\text {min }}^{2} / x^{2}, l_{\min } \sim l_{p}, 0<\alpha \leq 1 / 4$ where $x$ is the measuring scale, introduced by the author in a series of works[22-32] meets the above requirements.

Note that this parameter has been introduced to study the 
deformation of QFT at Planck's scale, although the deformation per se, associated with a high-energy modification of the density matrix, was "minimal" in that it presented no noncommutativity operators related to different spatial coordinates

$$
\left[X_{i}, X_{j}\right] \neq 0, i \neq j
$$

and hence "limited" as in the end it failed to lead to GUP. Nevertheless, the corresponding deformation parameter in some way is universal.

This paper continues the studies, described in[33-35] (the latter in particular), of the fundamental quantities in "thermodynamic interpretation" of gravity[16-21] for GUP and EUP deformations of the latter. Compared to the works[35], the results from which are used in this paper, the important results associated with EUP are put forward together with the demonstration that GUP and EUP have the same deformation parameter, at least in this context.

The structure of this work is as follows. In Section 2 it is shown that the deformation of the fundamental thermodynamic quantities for black holes within GUP and EUP may be interpreted with the use of the same parameter. In Section 3 , within the scope of a dynamic model for the cosmological constant $\Lambda$ (vacuum energy density), GUP is studied for the pair $(\Lambda, V)[33,34]$, where $V-$ is the "space-time volume". In this Section consideration is given to the possible existence of EUP for this pair, i.e. to a possible extension of the Uncertainty Principle to the pair in the IR region, and hence to the possible substantiation of the proper (coincident with the experimental) value for $\Lambda$. In Section 4 the results of Section 2 are applied to Einstein's Equations for space with horizon and to Friedmann's Equations. It is demonstrated that in both cases their deformation (in the first case within GUP and in the second case within EUP) may be interpreted with the use of the same small dimensionless parameter having a known variability domain.

And, finally, in Section 5 the problems of further investigations are discussed, some final comments are given.

\section{Universal Deformation Parameter in Gravitational Thermodynamics with GUP and EUP}

In this Section the Gravitational Thermodynamics (GT) is understood as thermodynamics of spaces with horizon[18, 20].

\subsection{Gravitational Thermodynamics with GUP}

We use the notation and principal results from[14]. So, GUP is of the form

$$
\Delta x_{i} \Delta p_{j} \geq \hbar \delta_{i j}\left[1+\alpha^{2} l_{p}^{2} \frac{\left(\Delta p_{i}\right)^{2}}{\hbar^{2}}\right]
$$

and, since $\Delta x_{i} \Delta p_{j}>\hbar \delta_{i j}$, we have

$$
\Delta x_{i} \Delta p_{i} \geq \hbar \delta_{i j}\left[1+\alpha^{\prime 2} \frac{l_{p}^{2}}{\Delta x_{i}^{2}} \frac{\left(\Delta p_{i}\right)^{2} \Delta x_{i}^{2}}{\hbar^{2}}\right]>\hbar \delta_{i j}\left[1+\frac{1}{4} \alpha_{\Delta x_{i}}\right]
$$

where $\alpha_{\Delta x}$-parameter $\alpha$ corresponding to $\Delta x_{i}, l_{\min }=2 \alpha^{\prime} l_{p}$. Besides, as distinct from[14], for the dimensionless factor in GUP, instead of $\alpha$, we use $\alpha^{\prime}$ to avoid confusion with the deformation parameter.

In this terms the uncertainty in moment is given by the nonstrict inequality

$$
\begin{aligned}
& 2 \hbar\left(\alpha_{\Delta x_{i}} \Delta x_{i}\right)^{-1}\left[1-\sqrt{1-\alpha_{\Delta x_{i}}}\right] \leq \Delta p_{i} \leq \\
& 2 \hbar\left(\alpha_{\Delta x_{i}} \Delta x_{i}\right)^{-1}\left[1+\sqrt{1-\alpha_{\Delta x_{i}}}\right] .
\end{aligned}
$$

But for the quantities determining GT in terms of $\alpha$ one can derive exact expressions. Indeed, in terms of $\alpha$ the GUP-modification (or rather GUP-deformation)is easily obtained for the Hawking temperature[36-39,14,15] that has been computed in the asymptotically flat $d$ - dimensional space for a Schwarzshild black hole with a metric given by

$$
d s^{2}=-N^{2} d t^{2}+N^{-2} d r^{3}+r^{2} d \Omega_{d-2}^{2},
$$

where

$$
N^{2}=1-\frac{16 \pi G M}{(d-2) \Omega_{d-2} r^{d-3}},
$$

$\Omega_{d-2}$ is the area of the unit sphere $S^{n-2}$, and $r_{+}$is the uncertainty in the emitted particle position by the Hawking effect, expressed as

$$
\Delta x_{i} \approx r_{+}
$$

and being nothing else but a radius of the event horizon. In this case the deformation parameter $\alpha$ arises naturally. Actually, modification of the Hawking temperature is of the form, see formula (10) in[14]

$$
T_{G U P}=\left(\frac{d-3}{4 \pi}\right) \frac{\hbar r_{+}}{2 \alpha^{2} l_{p}^{2}}\left[1-\left(1-\frac{4 \alpha^{2} l_{p}^{2}}{r_{+}^{2}}\right)^{1 / 2}\right]
$$

and may be written in a natural way as

$$
T_{G U P}=2\left(\frac{d-3}{4 \pi}\right) \frac{\hbar}{r_{+}} \alpha_{r_{+}}^{-1}\left[1-\left(1-\alpha_{r_{+}}\right)^{1 / 2}\right],
$$

where $\alpha_{r}$-parameter $\alpha$ associated with $r_{+}$. It is clear that $T_{\text {GUP }}$ is actually the deformation $T_{\text {Hawk }}$ - black hole temperature for a semiclassical case[40]. In such a manner compared to $T_{\text {Hawk }} T_{G U P}$ is additionally dependent only on the dimensionless small deformation parameter $\alpha_{r_{+}}$.

The dependence of the black hole entropy on $\alpha_{r_{+}}$may be derived in a similar way. For a semiclassical approximation of the Bekenstein-Hawking formula[41,40]

$$
S=\frac{1}{4} \frac{A}{l_{p}^{2}},
$$

where $A$ - surface area of the event horizon, provided the horizon event is of radius $r_{+}, A \sim r_{+}^{2}$ and (10) is clearly of the form

$$
S=\sigma \alpha_{r_{+}}^{-1},
$$

where $\sigma$ is some dimensionless denumerable factor. The general formula for quantum corrections [38] given as

$$
\begin{aligned}
& S_{G U P}=\frac{A}{4 l_{p}^{2}}-\frac{\pi \alpha^{\prime 2}}{4} \ln \left(\frac{A}{4 l_{p}^{2}}\right)+ \\
& \sum_{n=1}^{\infty} c_{n}\left(\frac{A}{4 l_{p}^{2}}\right)^{-n}+\text { const },
\end{aligned}
$$

where the expansion coefficients $c_{n} \propto \alpha^{2(n+1)}$ can always be 
computed to any desired order of accuracy[38], may be also written in the general case as a Laurent series in terms of $\alpha_{r_{t}}$

$$
\begin{aligned}
& S_{G U P}=\sigma \alpha_{r_{+}}^{-1}-\frac{\pi \alpha^{\prime 2}}{4} \ln \left(\sigma \alpha_{r_{+}}^{-1}\right) \\
& +\sum_{n=1}^{\infty}\left(c_{n} \sigma^{-n}\right) \alpha_{r_{+}}^{n}+\text { const. }
\end{aligned}
$$

In what follows the representation in terms of the deformation parameter $\alpha$ is referred to as $\alpha$-representation.

\section{Gravitational Thermodynamics with EUP}

Let us consider QFT with EUP[14]. In this case we obtain QFT with $p_{\min }$. Obviously, there is no minimal length $l_{\min }$ in QFT with EUP whatsoever but we assume that QFT with GUP is valid. At the present time for such an assumption we can find solid argumentation[6-9]. As will be shown later, in this case the fundamental quantities may be also expressed in terms of $\alpha$. Hereinafter we use a small dimensionless parameter

$$
\alpha_{i}=\frac{l_{o r}^{2}}{\tilde{l}^{2}},
$$

where $l_{o r} \equiv l_{\text {original }}=2 \alpha^{\prime} l_{p}, \alpha^{\prime}$-dimensionless constant on the order of unity from GUP (2), and it is suggested that

$$
2 l_{o r} \leq \tilde{l} \text {, i.e } 0<\alpha_{i} \leq 1 / 4 \text {. }
$$

Similar to the previous Section, it is convenient to use the principal results of[14] (sections 3,4). Then EUP in (A)dS space takes the form

$$
\Delta x_{i} \Delta p_{j} \geq \hbar \delta_{i j}\left[1+\beta^{2} \frac{\left(\Delta x_{i}\right)^{2}}{t^{2}}\right]
$$

where $l$ is the characteristic, large length scale $l \gg l_{p}$ and $\beta$ is a dimensionless real constant on the order of unity[14]. From EUP there is an absolute minimum in the momentum uncertainty:

$$
\Delta p_{i} \geq \frac{2 \hbar \beta}{l},
$$

and EUP (16) may be rewritten as

$$
\begin{aligned}
& \Delta x_{i} \Delta p_{j} \geq \hbar \delta_{i j}\left[1+\beta^{2} \frac{\left(\Delta x_{i}\right)^{2}}{t_{o r}^{2}} \frac{l_{o r}^{2}}{t^{2}}\right] \\
& =\hbar \delta_{i j}\left[1+\beta^{2} \alpha_{l} \alpha_{\Delta x_{i}}^{-1}\right] .
\end{aligned}
$$

Considering that in a theory with fixed $l \gg l_{p}$

$$
\alpha_{l}=\text { const } \ll 1,
$$

(16),(18) may be written as

$$
\Delta x_{i} \Delta p_{j} \geq \hbar \delta_{i j}\left[1+\beta^{2} \alpha_{l} \alpha_{\Delta x_{i}}^{-1}\right]=\hbar \delta_{i j}\left[1+\widetilde{\beta}^{2} \alpha_{\Delta x_{i}}^{-1}\right],
$$

where $\beta$ is redetermined as

$$
\beta \mapsto \widetilde{\beta}=\sqrt{\alpha_{l}} \beta .
$$

However, in this case $\beta$ may be left as it is, whereas $\alpha$ may be redetermined because $\alpha_{\Delta x_{i}}^{-1}$ in (18),(20) is not a small parameter. In consequence we can redeterminate $\alpha$ as

$$
\tilde{\alpha}_{\Delta x_{i}}=\alpha_{l} \alpha_{\Delta x_{i}}^{-1},
$$

where $\tilde{\alpha}_{\Delta_{i}}$ is now a small parameter.
Owing to such a duality, EUP (16), (18) may be rewritten in terms of a new small parameter $\alpha$ similar to $\alpha$ as follows:

$$
\Delta x_{i} \Delta p_{j} \geq \hbar \delta_{i j}\left[1+\beta^{2} \widetilde{\alpha}_{\Delta x_{i}}\right] .
$$

Then in analogy with [14] (Section 3), for Hawking temperature of the $d$-dimensional Schwarzshild-AdS black hole with the metric function we have

$$
N^{2}=1+\frac{r^{2}}{l_{\text {AdS }}^{2}}-\frac{16 \pi G M}{(d-2) \Omega_{d-2} r^{d-3}}
$$

in the metric of(5) and the cosmological constant $\Lambda=-(d-1)(d-2) / 2 l_{A d S}^{2}$.

Therewith the $\alpha$-representation of the Hawking temperature $T_{E U P}[14]$ (formula (15)) takes the form

$$
\begin{aligned}
& T_{E U P(A d S)}=\left(\frac{d-3}{4 \pi}\right) \frac{\hbar}{r_{+}^{2}}\left[1+\frac{(d-1)}{(d-3)} \alpha_{r_{+}}^{-1} \alpha_{l_{A d S}}\right] \\
& =\left(\frac{d-3}{4 \pi}\right) \frac{\hbar}{r_{+}^{2}}\left[1+\frac{(d-1)}{(d-3)} \widetilde{\alpha}_{r_{+}}\right] .
\end{aligned}
$$

In the same way we can easily obtain the $\alpha$ - representation of the Hawking temperature for a Schwarzshild-AdS black hole and for a combined case ((formula (28) from the[14])) of GUP and EUP - (GEUP)

$$
\begin{aligned}
& T_{G E U P(A d S)}= \\
& 2\left(\frac{d-3}{4 \pi}\right) \frac{\hbar}{r_{+}} \alpha_{r_{+}}^{-1}\left[1-\sqrt{1-\alpha_{r_{+}}\left[1+\frac{\alpha_{l_{A d s}}(d-1)}{(d-3)} \alpha_{r_{+}}^{-1}\right]}\right] \\
= & 2\left(\frac{d-3}{4 \pi}\right) \frac{\hbar}{r_{+}} \alpha_{r_{+}}^{-1}\left[1-\sqrt{\frac{d-3-\alpha_{l_{A d S}}(d-1)}{(d-3)}-\alpha_{r_{+}}}\right],
\end{aligned}
$$

i.e. in the general case we get a Laurent series from $\alpha$.

Similarly, we can obtain the $\alpha$-representation for the corresponding value of $T_{\text {GEUP(dS) }}$ ((formula (32) from[14]) in the de Sitter (dS) space by the substitution $l_{d d S}^{2} \rightarrow-l_{d s}^{2}$.

Note that, as it has been indicated in[33,34], $\alpha_{r_{+}}^{-1}$ has one more interesting feature

$$
\alpha_{r_{+}}^{-1} \sim r_{+}^{2} / l_{p}^{2} \sim S_{B H} .
$$

Here $S_{B H}$ is the Bekenstein-Hawking semiclassical black hole entropy with the characteristic linear size $r_{+}$. For example, in the spherically symmetric case $r_{+}=R$ - radius of the corresponding sphere with the surface area $A$, and

$$
A=4 \pi r_{+}^{2}, S_{B H}=A / 4 l_{p}^{2}=\frac{\pi}{4 \alpha^{\prime 2}} \alpha_{r_{+}}^{-1} .
$$

In [15] GUP and EUP are combined by the principle called the Symmetric Generalized Uncertainty Principle (SGUP):

$$
\Delta x \Delta p \geq \hbar\left(1+\frac{(\Delta x)^{2}}{L^{2}}+t^{2} \frac{(\Delta p)^{2}}{\hbar^{2}}\right),
$$

where $l \ll L$ and $l$ defines the limit of the UV-cutoff (not being such up to a constant factor as in the case of GUP).Then a minimal length is determined as

$$
\Delta x_{\min }=2 l / \sqrt{1-4 t^{2} / L^{2}},
$$

whereas $L$ defines the limit for IR-cutoff i. e. we have a minimum momentum

$$
\Delta p_{\text {min }}=2 \hbar /\left(L \sqrt{1-4 t^{2} / L^{2}}\right) .
$$


And using the Euclidian action formalism by Gibbons and Hawking[42], in[15] the corresponding correction of the Hawking temperature for an ordinary(not $\mathrm{A}(\mathrm{dS})$ ) Schwarzshild-black hole is computed. This correction is given as $T_{S G U P}$. In the notation of this work

$$
\Delta x_{\min }=2 l / \sqrt{1-4 \alpha_{l}^{-1} \alpha_{L}}=2 l / \sqrt{1-4 \widetilde{\alpha_{l}}},
$$

where $\widetilde{\alpha}_{l}$-small parameter introduced in conformity with (22). We can easily obtain the $\alpha$-representation for $T_{S G U P}$ that is completely similar to the $\alpha$-representation of $T_{\text {GEUP(AdS) }}$.

It should be noted that in the realistic theories $l \sim l_{p}$, and it is obvious that $\left(\sqrt{1-4 l^{2} / L^{2}}\right) \approx 1$. Thus, $\Delta x_{\min } \approx 2 l \sim 2 l_{p}$ and hence in this case we get a minimal length that is much the same (to within $\alpha$ ) as in the case of GUP. It is seen that, with due regard for the requirement $l \ll L, \Delta p_{\min }$ is derived close (to within $\beta$ ) to $\Delta p_{\min }(17)$ in a theory with EUP.

The question arises as to what for all these manipulations with writing and rewriting of the already derived expressions in the $\alpha$-representation are necessary.

1. Owing to this procedure, we can draw the conclusion that all the quantities within the scope of the stated problem are dependent on one and the same deformation parameter $\alpha$ that is small, dimensionless (discrete in the case of GUP), and varying over the given interval. And, provided the infrared cutoff $l$ is defined, we have

$$
\alpha_{l}=\alpha_{\text {min }}=l_{o r}^{2} / l^{2} \leq \alpha \leq 1 / 4 \text { and } l_{o r} \equiv l_{\text {original }} \sim l_{p} .
$$

If we primordially consider a theory with GUP only, then $l_{o r} \equiv l_{\text {min }}$. But in the arbitrary case it is required that $l_{o r}=2 \alpha^{\prime} l_{p}$, where $\alpha$ is a certain dimensionless constant on the order of unity.

The property of discreteness is retained for $\alpha$ in the cases when only GUP (without generalizations)is valid because in this case the length seems to be quantized, the lengths being considered from $2 l_{\text {min }}$ rather than from $l_{o r}=l_{\text {min }}$ as a singularity arises otherwise[23-30].

2. Actually, all the quantities may be represented as a Laurent series in terms of $\alpha$, and a solution of the problem at hand may be understood as finding of the members in this series.

3. When the problem has separate solutions for the cases including the UV- and IR-cutoffs, we can consider expansion in each of the cases in terms of their own small parameters: $\alpha$ in the case of UV-cutoff and $\alpha$ in the case of IR-cutoff, where $\alpha$ is a duality of $\alpha$

$$
\tilde{\alpha}_{i}=\alpha_{l} \alpha_{\tilde{l}}^{-1}
$$

$l$ determines, to within a factor on the order of unity, the characteristic system's size, and $l \gg l_{p}$.

\section{The Cosmological Constant Problem and QFT with GUP and SGUP}

In this section it is assumed that $\Lambda$ may be varying in time. Generally speaking, $\Lambda$ is referred to as a constant just because it is such in the equations, where it occurs: Einstein equations[43]. But in the last few years the dominating point of view has been that $\Lambda$ is actually a dynamic quantity, now weakly dependent on time[44-46]. It is assumed therewith that, despite the present-day smallness of $\Lambda$ or even its equality to zero, nothing points to the fact that this situation was characteristics for the early Universe as well. Some recent results[47-50] are rather important pointing to a potentially dynamic character of $\Lambda$. Specifically, of great interest is the Uncertainty Principle derived in these works for the pair of conjugate variables $(\Lambda, V)$ :

$$
\Delta \Lambda \Delta V \sim \hbar
$$

where $\Lambda$ is the vacuum energy density (cosmological constant). It is a dynamic value fluctuating around zero; $V$ is the space-time volume. Here the volume of space-time $V$ results from the Einstein-Hilbert action $S_{E H}$ [48]:

$$
\Lambda \int d^{4} x \sqrt{-g}=\Lambda V
$$

where (31) is the term in the $S_{E H}$. In this case the notion of conjugation is well-defined, but approximate, as implied by the expansion about the static Fubini-Study metric (Section 6.1 of [47]). Unfortunately, in the proof per se (30), relying on the procedure with a non-linear and non-local Wheelerde-Witt-like equation of the background-independent Matrix theory, some unconvincing arguments are used, making it insufficiently rigorous (Appendix 3 of[47]). But, without doubt, this proof has a significant result, though failing to clear up the situation.

In $[33,34,51]$ the Heisenberg Uncertainty Relation for the pair $(\Lambda, V)(30)$ has been generalized to GUP

$$
\Delta V \geq \frac{\hbar}{\Delta \Lambda}+\alpha_{\Lambda}^{\prime} t_{p}^{2} \bar{V}_{p}^{2} \frac{\Delta \Lambda}{\hbar}
$$

or that is the same

$$
\Delta V \Delta \Lambda \geq \hbar\left(1+\alpha_{\Lambda}^{\prime} t_{p}^{2} \bar{V}_{p}^{2} \frac{(\Delta \Lambda)^{2}}{\hbar^{2}}\right) .
$$

where $\alpha_{\Lambda}^{\prime}$ is a new constant and $\bar{V}_{p}=l_{p}^{3}$.

In the case of UV - limit: $t \rightarrow t_{\min }, \Delta \Lambda$ becomes significant

$$
\begin{aligned}
& \lim _{t \rightarrow t_{\text {min }}} \bar{V}=\bar{V}_{\text {min }} \sim \bar{V}_{p}=l_{p}^{3} ; \\
& \lim _{t \rightarrow t_{\min }} V=V_{\text {min }} \sim V_{p}=l_{p}^{3} t_{p},
\end{aligned}
$$

where $V$ - spatial part of $V$

The existence of $V_{\min } \sim V_{p}$ directly follows from GUP for the pair $(p, x)(2)$ and GUP for the pair $(E, t)[24,30]$ as well as from solutions of the quadratic inequalities(32),(33).

So, (32) is nothing else but

$$
\Delta V \geq \frac{\hbar}{\Delta \Lambda}+\alpha_{\Lambda}^{\prime} V_{p}^{2} \frac{\Delta \Lambda}{\hbar} .
$$

And in the case of UV - cutoff we have

$$
\lim _{t \rightarrow t_{\min }} \Lambda \equiv \Lambda_{U V} \sim \Lambda_{p} \equiv \hbar / V_{p}=E_{p} / \bar{V}_{p} .
$$

It is easily seen that in this case $\Lambda_{U V} \sim m_{p}^{4}$, in agreement with the value obtained using a standard (i.e. without super-symmetry and the like) quantum field theory[52,53]. Despite the fact that $\Lambda$ at Planck's scales (referred to as $\Lambda_{U V}$ ) is also a dynamic quantity, it is not directly related to the familiar $\Lambda$ because the latter, as opposed to the first one, 
is derived from Einstein's equations

$$
R_{\mu \nu}-\frac{1}{2} g_{\mu v} R=8 \pi G_{N}\left(-\Lambda g_{\mu v}+T_{\mu v}\right) .
$$

However, Einstein's equations (37) are not valid at the Planck scales and hence $\Lambda_{U V}$ may be considered as some high-energy generalization (deformation) of the conventional cosmological constant in the low-energy limit.

The problem is whether a correct generalization of GUP for the pair $(\Lambda, V)(33)$ to the Symmetric Generalized Uncertainty Principle (SGUP) of the form given by (29) is possible. If the answer is positive, a theory also includes $\Lambda_{\text {min }}$ that may be referred to as $\Lambda_{I R}$ in similarity with $\Lambda_{U V}$. Then, similar to $T$ (29), an additional term defining the IR-cutoff must be of the form

$$
\Omega_{I R}=\frac{(\Delta V)^{2}}{\widetilde{V}^{2}},
$$

where $\widetilde{V}$ - certain space-time volume effectively specifying the IR-limit of the observable part of the Universe with the spatial part $\tilde{\bar{V}} \sim L^{3} ; L-$ radius of the observable part of the Universe. Now it is known that $L \approx 10^{28}$. Clearly, the introduction of an additional term of the form (38) into the right-hand side of (33) leads to $\Lambda_{I R} \ll \Lambda_{U V}$ and might lead to the value of $\Lambda$ close to the experimental value $\Lambda_{\text {exp }}$ [54]. Note that the Holographic Principle[55-61] used to the Universe as a whole[61] gives $\Lambda_{\text {exp }}[60]$. In[62,63,33,34] it has been demonstrated that the $\alpha$-representation ( $\alpha$ -deformation) of QFT with GUP plays a significant role. In particular, consider

$$
\Lambda_{\text {exp }} \approx \alpha_{L} \Lambda_{U V},
$$

where $L \approx 10^{28}$.(39) is like (22). But the Holographic Principle imposes strict restrictions on the number of degrees of freedom in the Universe, and hence for us it is important to study the inferences of introducing the additional term of the form (38) in (33).

\section{GUP, EUP, and General Relativity Deformation}

In this Section we use the previously obtained results for some cases of high- energy and low-energy deformation of GR. Specifically, we demonstrate that in the cases when the Thermodynamics Approach[16-21] is applicable to the General Relativity the deformation of GR with GUP and EUP may be a natural result of the $\alpha$-representation.

\section{1. $\alpha$-Representation of Einstein's Equations for Space with Horizon}

Let us consider $\alpha$-representation and high energy $\alpha$ deformation of the Einstein's field equations for the specific cases of horizon spaces (the point (c) of Section 4). In so doing the results of the survey work ([20] p.p.41,42)are used. Then, specifically, for a static, spherically symmetric horizon in space-time described by the metric

$$
d s^{2}=-f(r) c^{2} d t^{2}+f^{-1}(r) d r^{2}+r^{2} d \Omega^{2}
$$

the horizon location will be given by simple zero of the function $f(r)$, at $r=a$.

It is known that for horizon spaces one can introduce the temperature that can be identified with an analytic continuation to imaginary time. In the case under consideration ([20], eq.(116))

$$
k_{B} T=\frac{\hbar c f^{\prime}(a)}{4 \pi} .
$$

Therewith, the condition $f(a)=0$ and $f^{\prime}(a) \neq 0$ must be fulfilled.

Then at the horizon $r=a$ Einstein's field equations

$$
\frac{c^{4}}{G}\left[\frac{1}{2} f^{\prime}(a) a-\frac{1}{2}\right]=4 \pi P a^{2}
$$

may be written as the thermodynamic identity ([20] formula (119))

$$
\begin{aligned}
& \underbrace{\frac{\hbar c f^{\prime}(a)}{4 \pi} \frac{c^{3}}{G \hbar} d\left(\frac{1}{4} 4 \pi a^{2}\right)}_{k_{B} T} \underbrace{-\frac{1}{2} \frac{c^{4} d a}{G}}_{d S} \\
& =\underbrace{P d\left(\frac{4 \pi}{3} a^{3}\right)}_{-d E}
\end{aligned}
$$

where $P=T_{r}^{r}$ is the trace of the momentum-energy tensor and radial pressure. In the last equation $d a$ arises in the infinitesimal consideration of Einstein's equations when studying two horizons distinguished by this infinitesimal quantity $a$ and $a+d a$ ([20] formula (118)).

Now we consider (43) in a new notation, expressing $a$ in terms of the corresponding deformation parameter $a$. Hereinafter in this Section we write $a$ instead of $\alpha_{a}$ as we consider the same $a$. Then we have

$$
a=l_{\text {min }} \alpha^{-1 / 2} \text {. }
$$

Therefore,

$$
f^{\prime}(a)=-2 l_{\min }^{-1} \alpha^{3 / 2} f^{\prime}(\alpha) .
$$

Substituting this into (42) or into (43), we obtain in the considered case of Einstein's equations in the " $a$ -representation" the following:

$$
\frac{c^{4}}{G}\left(-\alpha f^{\prime}(\alpha)-\frac{1}{2}\right)=4 \pi P \alpha^{-1} l_{\min }^{2} .
$$

Multiplying the left- and right-hand sides of the last equation by $a$, we get

$$
\frac{c^{4}}{G}\left(-\alpha^{2} f^{\prime}(\alpha)-\frac{1}{2} \alpha\right)=4 \pi P l_{\text {min }}^{2} .
$$

But since usually $l_{\text {min }} \sim l_{p}$ (that is just the case if the Generalized Uncertainty Principle (GUP) is satisfied), we have $l_{\min }^{2} \sim l_{p}^{2}=G \hbar / c^{3}$. When selecting a system of units, where $\hbar=c=1$, we arrive at $l_{\text {min }} \sim l_{p}=\sqrt{ } G$, and then (46) is of the form

$$
-\alpha^{2} f^{\prime}(\alpha)-\frac{1}{2} \alpha=4 \pi P \vartheta^{2} G^{2},
$$

where $\vartheta=l_{\min } / l_{p}$. L. h. s. of (48) is dependent on $a$. Because of this, r. h. s. of (48) must be dependent on $a$ as well, 
i. e. $P=P(\alpha)$.

Analysis of $a$-Representation of Einstein's Equations

Now let us get back to (43). In[20] the low-energy case has been considered, for which ([20] p.42 formula (120))

$$
S=\frac{1}{4 l_{p}^{2}}\left(4 \pi a^{2}\right)=\frac{1}{4} \frac{A_{H}}{l_{p}^{2}} ; \quad E=\frac{c^{4}}{2 G} a=\frac{c^{4}}{G}\left(\frac{A_{H}}{16 \pi}\right)^{1 / 2},
$$

where $A_{H}$ is the horizon area. In our notation (49) may be rewritten as

$$
S=\frac{1}{4} \pi \alpha^{-1} ; \quad E=\frac{c^{4}}{2 G} a=\frac{c^{4}}{G}\left(\frac{A_{H}}{16 \pi}\right)^{1 / 2}=\frac{\vartheta}{2 \sqrt{ } G} \alpha^{1 / 2} \text {. }
$$

We proceed to two entirely different cases: low energy (LE) case and high energy (HE) case. In our notation these are respectively given by

$$
\text { A) } \alpha \rightarrow 0 \text { (LE), B) } \alpha \rightarrow 1 / 4 \text { (HE) }
$$

C) $a$ complies with the familiar scales and energies.

The case of C) is of no particular importance as it may be considered within the scope of the conventional General Relativity.

Indeed, in point A) $\alpha \rightarrow 0$ is not actually an exact limit as a real scale of the Universe (Infrared (IR)-cutoff $l_{\text {max }} \approx 10^{28} \mathrm{~cm}$ ), and then

$$
\alpha_{\min } \sim l_{p}^{2} / l_{\max }^{2} \approx 10^{-122}
$$

In this way A) is replaced by A1) $\alpha \rightarrow \alpha_{\min }$. In any case at low energies the second term in the left-hand side (48) may be neglected in the infrared limit. Consequently, at low energies (48) is written as

$$
-\alpha^{2} f^{\prime}(\alpha)=4 \pi P(\alpha) \vartheta^{2} G^{2} .
$$

Solution of the corresponding Einstein equation - finding of the function $f(\alpha)=f[P(\alpha)]$ satisfying (51). In this case formulae (49) are valid as at low energies a semiclassical approximation is true. But from (51)it follows that

$$
f(\alpha)=-4 \pi \vartheta^{2} G^{2} \int \frac{P(\alpha)}{\alpha^{2}} d \alpha .
$$

On the contrary, knowing $f(\alpha)$, we can obtain $P(\alpha)=T_{r}^{r}$.

Possible High Energy $a$-Deformation of General Relativity

Let us consider the high-energy case B). Here two variants are possible.

I. First variant.

In this case it is assumed that in the high-energy (Ultraviolet (UV))limit the thermodynamic identity (43) is retained but now all the quantities involved in this identity become $a$-deformed. This means that they appear in the $a$ -representation with quantum corrections and are considered at high values of the parameter $a$, i.e. at $a$ close to $1 / 4$. In particular, the temperature $T$ from equation (43) is changed by $T_{G U P}$ (9), the entropy $S$ from the same equation given by semiclassical formula (49) is changed by $S_{G U P}$ (13), and so forth:

$$
E \mapsto E_{G U P}, V \mapsto V_{G U P} .
$$

Then the high-energy $a$-deformation of equation (43) takes the form

$$
\begin{aligned}
& k_{B} T_{G U P}(\alpha) d S_{G U P}(\alpha)-d E_{G U P}(\alpha) \\
& =P(\alpha) d V_{G U P}(\alpha) .
\end{aligned}
$$

Substituting into (53) the corresponding quantities

$T_{G U P}(\alpha), S_{G U P}(\alpha), E_{G U P}(\alpha), V_{G U P}(\alpha), P(\alpha)$ and expanding them into a Laurent series in terms of $a$, close to high values of $a$, specifically close to $\alpha=1 / 4$, we can derive a solution for the high energy $a$-deformation of general relativity (53) as a function of $P(\alpha)$. As this takes place, provided at high energies the generalization of (43) to (53) is possible, we can have the high-energy $a$-deformation of the metric. Actually, as from (43) it follows that

$$
f^{\prime}(a)=\frac{4 \pi k_{B}}{\hbar c} T=4 \pi k_{B} T
$$

(considering that we have assumed $\hbar=c=1$ ), we get

$$
{f^{\prime}}_{G U P}(a)=4 \pi k_{B} T_{G U P}(\alpha) .
$$

L.h.s. of (55) is directly obtained in the $a$-representation. This means that, when $f^{\prime} \sim T$, we have $f^{\prime}{ }_{G U P} \sim T_{G U P}$ with the same factor of proportionality. In this case the function $f_{G U P}$ determining the high-energy $a$-deformation of the spherically symmetric metric may be in fact derived by the expansion of $T_{G U P}$, that is known from (9), into a Laurent series in terms of $a$ close to high values of $a$ (specifically close to $\alpha=1 / 4$ ), and by the subsequent integration.

It might be well to remark on the following.

1.1 As on going to high energies we use (GUP), $\vartheta$ from equation (48)is expressed in terms of $a$-dimensionless constant from GUP (2): $\vartheta=2 \alpha^{\prime}$.

1.2 Of course, in all the formulae including $l_{p}$ this quantity must be changed by $G^{1 / 2}$ and hence $l_{\text {min }}$ by $\vartheta G^{1 / 2}=2 \alpha^{\prime} G^{1 / 2}$.

1.3 As noted in the end of subsection 6.1, and in this case also knowing all the high-energy deformed quantities $T_{G U P}(\alpha), S_{G U P}(\alpha), E_{G U P}(\alpha), V_{G U P}(\alpha)$, we can find $P(\alpha)$ at $a$ close to $1 / 4$.

1.4 Here it is implicitly understood that the Ultraviolet limit of Einstein's equations is independent of the starting horizon space. This assumption is quite reasonable. Because of this, we use the well-known formulae for the modification of thermodynamics and statistical mechanics of black holes in the presence of GUP $[36-39,14,15]$.

1.5 The use of the thermodynamic identity (53) for the description of the high energy deformation in General Relativity implies that on going to the UV-limit of Einstein's equations for horizon spaces in the thermodynamic representation (consideration) we are trying to remain within the scope of equilibrium statistical mechanics[64] (equilibrium thermodynamics)[65]. However, such an assumption seems to be too strong. But some grounds to think so may be found as well. Among other things, of interest is the result from[36] that GUP may prevent black holes from their total evaporation. In this case the Planck's remnants of black holes will be stable, and when they are considered, in some approximation the equilibrium thermodynamics should be valid. At the same time, by author's opinion these arguments are rather weak to think that the quantum gravitational effects in this 
context have been described only within the scope of equilibrium thermodynamics[65].

II. Second variant.

According to the remark of 4.1.5, it is assumed that the interpretation of Einstein's equations as a thermodynamic identity (43) is not retained on going to high energies (UV-limit), i.e. at $\alpha \rightarrow 1 / 4$, and the situation is adequately described exclusively by non-equilibrium thermodynamics $[65,66]$. Naturally, the question arises: which of the additional terms introduced in (43) at high energies may be leading to such a description?

In the $[51,33]$ it has been shown that in case the cosmological term $\Lambda$ is a dynamic quantity, it is small at low energies and may be sufficiently large at high energies. In the right-hand side of (48) in the $a$-representation the additional term $G F(\Lambda(\alpha))$ is introduced:

$$
-\alpha^{2} f^{\prime}(\alpha)-\frac{1}{2} \alpha=4 \pi P \vartheta^{2} G^{2}-G F(\Lambda(\alpha))
$$

where in terms of $F(\Lambda(\alpha))$ we denote the term including $\Lambda(\alpha)$ as a factor. Then its inclusion in the low-energy case (42)(or in the $a$-representation (48)) has actually no effect on the thermodynamic identity (43)validity, and consideration within the scope of equilibrium thermodynamics still holds true. It is well known that this is not the case at high energies as the $\Lambda$-term may contribute significantly to make the "process" non-equilibrium in the end $[65,66]$.

Is this the only cause for violation of the thermodynamic identity (43) as an interpretation of the high-energy generalization of Einstein's equations? Further investigations are required to answer this question.

\section{2. $\alpha$-Representation for Friedmann Equations with GUP and EUP}

Thermodynamic interpretation of Section 4 has been also developed for Friedmann Equations (FEs) of the FriedmannRobertson-Walker (FRW) Universe in[21]. In the process it is taken into consideration that in the FRW space-time, where the metric is given by the formula

$$
d s^{2}=-d t^{2}+a^{2}\left(\frac{d r^{2}}{1-k r^{2}}+r^{2} d \Omega_{n-1}^{2}\right),
$$

and $d \Omega_{n-1}^{2}$ denotes a line element of the (n-1)-dimensional unit sphere, $a$ is the scale factor, $k$ is the spatial curvature constant, there is a dynamic apparent horizon, the radius of which is as follows:

$$
\tilde{r}_{A}=\frac{1}{\sqrt{H^{2}+k / a^{2}}},
$$

where $H \equiv \dot{a} / a$ is the Hubble parameter.

FEs in[21] have been derived proceeding from the assumption that apparent horizon is endowed with the associated entropy and temperature such the event horizon in the black hole case

$$
S=\frac{A}{4 G}, \quad T=\frac{1}{2 \pi \tilde{r}_{A}},
$$

and from the validity of the first low of thermodynamics, we have

$$
d E=T d S .
$$

In[67] with the use of this thermodynamic interpretation of FEs the modifications of GUP and EUP (or more precisely the GUP and EUP deformations) of FEs have been obtained. It is clear that these (GUP and EUP)-deformed FEs may be written in the form of the $a$-representation. For simplicity, let us consider the case $n=3$.

Then for GUP the formula (26) from[67] takes the form

$$
\begin{aligned}
& \left(\dot{H}-\frac{k}{a}\right)\left[1+\pi \alpha^{\prime 2} l_{p}^{2} \frac{1}{A}+2\left(\pi \alpha^{\prime 2} l_{p}^{2}\right)^{2} \frac{1}{A^{2}}\right. \\
& \left.+\sum_{d=3} c_{d}\left(4 \pi \alpha^{\prime 2} l_{p}^{2}\right)^{2 d} \frac{1}{A^{d}}\right]=-4 \pi G(\rho+p),
\end{aligned}
$$

whereas in the $a$-representation its form is more elegant

$$
\begin{aligned}
& \left(\dot{H}-\frac{k}{a}\right)\left[1+\frac{1}{16} \alpha_{\tilde{r}_{A}}+\frac{1}{32} \alpha_{\tilde{r}_{A}}^{2}\right. \\
& \left.+\sum_{d=3} \frac{c_{d}}{4^{d}} \alpha_{\tilde{r}_{A}}^{d}\right]=-4 \pi G(\rho+p),
\end{aligned}
$$

Also, more elegant is $a$-representation of the second Friedmann Equation (formula (27) from [67])

$$
\begin{gathered}
\frac{8 \pi G^{2}}{3} \rho= \\
\qquad \frac{\alpha_{\tilde{r}_{A}}}{4 \pi \alpha^{\prime 2}}\left[\pi+\frac{1}{32} \alpha_{\tilde{r}_{A}}+\frac{1}{96} \alpha_{\tilde{r}_{A}}^{2}\right. \\
\left.\backslash+\sum_{d=3} \frac{c_{d}}{4^{d}(d+1)} \alpha_{\tilde{r}_{A}}^{d}\right],
\end{gathered}
$$

with the assumption that $\hbar=c=1$.

It is obvious that therewith familiar FEs appear at low energies, i.e. at $\alpha_{\tilde{r}_{A}} \ll 1 / 4$.

In the nontrivial high-energy case one can obtain the solution for FE and, in particular $\rho, H, p$ as a series in terms of a close to $1 / 4$.

In the case of EUP the $a$-representation of the deformed FE[67] seems to be even simpler. Specifically, using (18) (23), one can derive deformed first Friedmann equation of the form

$$
\begin{gathered}
\left(\dot{H}-\frac{k}{a^{2}}\right)\left(1+\frac{\beta^{2}}{\pi l^{2}} A\right)=\left(\dot{H}-\frac{k}{a^{2}}\right)\left(1+\frac{\beta^{2}}{\pi l^{2}} \frac{4 \pi \tilde{r}_{A}^{2}}{l_{o r}^{2}} l_{o r}^{2}\right) \\
=\left(\dot{H}-\frac{k}{a^{2}}\right)\left(1+4 \beta^{2} \alpha_{\tilde{r}_{A}}^{-1} \alpha_{l_{o r}}\right) \\
=\left(\dot{H}-\frac{k}{a^{2}}\right)\left(1+4 \beta^{2} \widetilde{\alpha}_{\tilde{r}_{A}}\right)=-4 \pi G(\rho+p),
\end{gathered}
$$

where, as expected, the deformation parameter $\tilde{\alpha}_{\tilde{r}_{A}}$ is small.

In a similar way we can obtain the $a$-representation of the EUP-deformation for the second Friedmann equation.

\section{Some Comments and Problems of Interest}

In this Section some comments are given and some problems are stated.

C1. The Laurent series expansion in terms of $a$ is asymmetric for UV and IR cutoffs. Indeed, as in the general case the variability domain $0<\alpha \leq 1 / 4$, in the UV-cutoff 
when $\alpha \approx 1 / 4$ the contribution is made by $a$-terms both with positive and with negative powers, while in the IR-cutoff $(\alpha \ll 1 / 4)$ only the $a$-terms with negative powers will be significant.

$\mathrm{C} 2$. The external constant $a$ in the cases, where $l_{o r} \neq l_{\min }$ (EUP or SGUP is the case), is not found in the final expressions, being reduced due to the substitution of (22).

Several questions remain to be answered and necessitate further investigations.

Q1) How far the $a$-representation may be extended for the General Relativity? As shown in this work, such a representation exists for the General Relativity at High and Low Energies when the Thermodynamic Approach[16-21] is applicable or, that is the same, the Thermodynamic Interpretation is the case. It is interesting whether the extension of the $a$-representation to the general case both at High and Low Energies is possible. The problem is whether, in some or other way, the general case may be reduced to the well-known ones.

Q2) Considering Q1), for High Energy the problem is whether there is an effective description of the space-time foam [68-70] in terms of $a$. The results of[71] suggest that such a description should be existent.

Q3) Proceeding from the results of E. Verlinde[72], the problem is whether the High-Energy deformation of the Entropic Force is obtainable. Provided the answer of Q1) positive, the problem concerns the form of this deformation in terms of $a$ : we must find its $a$-representation.

Note that the notion of Entropic Force, however, without the introduction of the term per se has been proposed by $\mathrm{T}$. Padmanabhan in Conclusion of his paper[73] earlier than by E. Verlinde.

\section{Conclusions}

The essence of the principal result obtained in this work may be stated as follows.

In the cases when Einstein equations have an explicit thermodynamic interpretation, their limiting transition to $U V$ and IR cutoffs may be described within the scope of the same dimensionless deformation parameter that is naturally introduced in a quantum field theory with the fundamental length.

In the case the problems stated in the previous Section will be solved positively, the small dimensionless discrete parameter $a$ must be at once introduced in High-Energy Thermodynamics and Gravity, without its appearance in the low-energy limit at the scales under study. At the same time, at large scales GR has not been subjected to verification too [74]. The availability of Dark Matter and Dark Energy is a strong motivation for the IR-modification of GR [75-77]. The deformation of the General Relativity due to EUP seems to be one of the IR-modifications of Gravity possible. In this case an analysis of such a deformation in terms of the parameter $a$, of the corresponding variability domain, and the like may be important for studies of the IR-modified
(IR-deformed) General Relativity.

\section{ACKNOWLEDGMENTS}

I am grateful to Prof. Sabine Hossenfelder (Stockholm, Sweden) for the information about a number of interesting works in the field of physics at Planck's scales and for her support that has stimulated my research efforts. Besides, I would like to thank Prof. Rong-Jia Yang (Baoding, China) for his support.

\section{REFERENCES}

[1] Klinkhamer F.R., 2007, Fundamental length scale of quantum spacetime foam, .JETPLett. 86, 2167-2180.

[2] Amelino-Camelia G., and Smolin L., 2009,Prospects for constraining quantum gravity dispersion with near term observations. Phys. Rev. D 80, 084017;Gubitosi G. et al., 2009,A Constraint on Planck-scale Modifications to Electrodynamics with CMB polarization data. JCAP 0908, 021; Amelino-Camelia G., 2005, Building a case for a Planck-scale-deformed boost action: the Planck-scale particle-localization limit. Int.J.Mod.Phys.D 14, 2167-2180.

[3] Hossenfelder S. et al., 2003, Signatures in the Planck Regime. Phys. Lett. B ,575, 85-99; Hossenfelder S., 2004, Running coupling with minimal length Phys. Rev. D ,70, 105003; Hossenfelder S., 2006,Self-consistency in theories with a minimal length, Class. Quant. Grav., 23, 1815-1821.

[4] Faddeev, L., 1989, Mathematical View on Evolution of Physics. Priroda, 5, 11-18.

[5] Veneziano, G. A., 1986, Stringy Nature Needs Just Two Constants Europhys.Lett, 2, 199-211; Amati, D.; Ciafaloni, M., and Veneziano, G., 1989,Can Space-Time Be Probed Below the String Size? Phys.Lett.B, 216, 41-47; E. Witten, Phys.Today 1996, 49, 24-28.

[6] Adler, R. J., and Santiago,D. I.,1999, On gravity and the uncertainty principle.Mod. Phys. Lett. A, 14, 1371-1378.

[7] Scardigli, F., 1999, Generalized uncertainty principle in quantum gravity from micro - black hole Gedanken experiment. Phys. Lett. B, 452, 39-44; Bambi, C. A., 2008, Revision of the Generalized Uncertainty Principle. Class. Quant. Grav, 25, 105003.

[8] Garay,L. Quantum gravity and minimum length. Int.J. Mod. Phys. A 1995, 10, 145-166.

[9] Ahluwalia,D.V., 2000, Wave particle duality at the Planck scale: Freezing of neutrino oscillations. Phys.Lett, A275, 31-35; Ahluwalia, D.V., 2002, Interface of gravitational and quantum realms., Mod.Phys.Lett, A17, 1135-1146.

[10] Maggiore,M. A., B304,Generalized uncertainty principle in quantum gravity. Phys.Lett, B304, 65-69.

[11] Maggiore,M.,1993, The Algebraic structure of the generalized uncertainty principle. Phys.Lett.B,304, 319, 83-86.

[12] Bolen, B., and Cavaglia, M., 2005, (Anti-)de Sitter black hole thermodynamics and the generalized uncertainty principle. Gen.Rel.Grav. , 37, 1255-1263. 
[13] Kempf,A., Mangano,G., Mann,R.B., 1995, Hilbert space representation of the minimal length uncertainty relation. Phys.Rev.D, 52, 1108-1118.

[14] Park, M.-I. 2008,, The Generalized Uncertainty Principle in (A)dS Space and the Modification of Hawking Temperature from the Minimal Length. Phys.Lett.B, 659, 698-702.

[15] Kim,Wontae., and Son,Edwin J.; Yoon, 2008, Myungseok. Thermodynamics of a black hole based on a generalized uncertainty principle. JHEP, 08, 035.

[16] Jacobson,T., 1995, Thermodynamics of space-time: The Einstein equation of state. Phys. Rev. Lett, 75, 1260-1263.

[17] Padmanabhan,T.,2002, The Holography of gravity encoded in a relation between entropy, horizon area and action for gravity. Gen.Rel.Grav ,34, 2029-2035.

[18] Padmanabhan,T. 2005, Gravity and the thermodynamics of horizons. Phys.Rept, 406, 49-125.

[19] Paranjape,A., Sarkar, S., Padmanabhan,T., 2006, Thermodynamic route to field equations in Lancos-Lovelock gravity. Phys.Rev. D, 2006, 74, 104015.

[20] Padmanabhan,T., 2010, Thermodynamical Aspects of Gravity: New insights.Rep. Prog. Phys., 74, 046901. arXiv:0911.5004, 22p.p.

[21] Cai,R.-G., and Kim,S.P., 2005, First law of thermodynamics and Friedmann equations of Friedmann-Robertson-Walker universe, JHEP, 02, 050.

[22] Shalyt-Margolin, A.E., and Suarez, J.G.,2003, Quantum mechanics of the early universe and its limiting transition. gr-qc/0302119, 16pp.

[23] Shalyt-Margolin, A.E., and Suarez, J.G., 2003, Quantum mechanics at Planck's scale and density matrix. Intern. Journ. Mod. Phys D, 12, 1265-1278.

[24] Shalyt-Margolin, A.E., and Tregubovich, A.Ya., 2004, Deformed density matrix and generalized uncertainty relation in thermodynamics. Mod. Phys.Lett. A, 19, 71-82.

[25] Shalyt-Margolin, A.E., 2004, Nonunitary and unitary transitions in generalized quantum mechanics, new small parameter and information problem solving. Mod. Phys. Lett. A, 19, 391-404.

[26] Shalyt-Margolin, A.E., 2004, Pure states, mixed states and Hawking problem in generalized quantum mechanics. Mod. Phys. Lett. A, 19, 2037-2045.

[27] Shalyt-Margolin, A.E., 2004, The Universe as a nonuniform lattice in finite volume hypercube. I. Fundamental definitions and particular featuresIntern. Journ. Mod.Phys D, 13, $853-864$

[28] Shalyt-Margolin, A.E.,2005, The Universe as a nonuniform lattice in the finitedimensional hypercube. II. Simple cases of symmetry breakdown and restoration. Intern.Journ. Mod. Phys. A, 20, 4951-4964.

[29] Shalyt-Margolin, A.E., and Strazhev,V.I, 2006, The Density Matrix Deformation in Quantum and Statistical Mechanics in Early Universe. In Proc. Sixth International Symposium "Frontiers of Fundamental and Computational Physics", edited by B.G. Sidharth at al. Springer,2006, pp.131-134.
[30] Shalyt-Margolin, A.E.,2005, The Density matrix deformation in physics of the early universe and some of its implications. In Quantum Cosmology Research Trends, edited by A. Reimer, Horizons in World Physics. 246, Nova Science Publishers, Inc., Hauppauge, NY,2005, pp. 49-91.

[31] Shalyt-Margolin, A.E.,2006, Deformed density matrix and quantum entropy of the black hole. Entropy, 8, 31-43.

[32] Shalyt-Margolin, A.E.,2007, Entropy in the Present and Early Universe. Symmetry, 18 , 299-320.

[33] Shalyt-Margolin, A.E.,2010, Entropy in the Present and Early Universe and Vacuum Energy, AIP Conference Proceedings, 1205, 160-167.

[34] Shalyt-Margolin, A.E.,2010, Entropy In The Present And Early Universe: New Small Parameters And Dark Energy Problem Entropy, 12, 932-952

[35] Shalyt-Margolin, A.E.,2010, Deformed Quantum Field Theory, Thermodynamics at Low and High Energies, and Gravity. I arXiv: 1003.4523, 23pp.

[36] Adler,R. J., Chen,P., Santiago, D. I.,2010, The generalized uncertainty principle and black hole remnants. Gen.Rel.Grav., 13, 2101-2108.

[37] Custodio, P. S., and Horvath, J. E.,2003, The Generalized uncertainty principle, entropy bounds and black hole (non)evaporation in a thermal bath. Class.Quant.Grav., 20, L197-L203.

[38] Medved, A.J.M., and Vagenas, E.C.,2004, When conceptual worlds collide: The GUP and the BH entropy. Phys. Rev. D, 70,124021 .

[39] Nouicer,K.,2007, Quantum-corrected black hole thermodynamics to all orders in the Planck length. Phys.Lett B, 646, 63-71.

[40] Hawking, S.,1976, Black Holes and Thermodynamics. Phys. Rev. D, 13,191-204.

[41] Bekenstein,J.D.,1973, Black Holes and Entropy. Phys.Rev.D, 7, 2333-2345.

[42] Gibbons, G.W., and Hawking, S. W., 1977, Action integrals and partition functions in quantum gravity, Phys.Rev. D, 15, 2752--2769.

[43] Wald R., 1984, General Relativity The University of Chicago Press Chicago and London 1984, 491 pp.

[44] Mukohyama, S., and Randall, L.,2004, A Dynamical approach to the cosmological constant. Phys.Rev.Lett, 92, 211302.

[45] Cai,Rong-Gen, and Hu,Bin, Zhang,Yi, 2009, Holography, UV/IR Relation, Causal Entropy Bound and Dark Energy. Commun. Theor. Phys., 51, 954-960.

[46] Shapiro, Ilya L.,and Sola, Joan.,2008, Can the cosmological "constant" run? - It may run. arXiv:0808.0315, 35pp.

[47] Jejjala, V., Kavic, M., Minic, D.,2007, Time and M-theory. Int. J. Mod. Phys. A ,22, 3317-3405.

[48] Jejjala, V., Kavic, M., Minic, D.,2007, Fine structure of dark energy and new physics., Adv. High Energy Phys, 2007, 21586. 
[49] Jejjala, V. and Minic, D.,2007, Why there is something so close to nothing: Towards a fundamental theory of the cosmological constant., Int.J.Mod.Phys.A ,22, 1797-1818.

[50] Jejjala, V., Minic, D.,Tze, C-H., 2004, Toward a background independent quantum theory of gravity. Int. J. Mod. Phys. D, $13,2307-2314$

[51] Shalyt-Margolin, A.E.,2009, Some Comments on Dynamical Character of Cosmological Constant and GUP, Foundations and Advances in Nonlinear Science, Proc. of the 14th Conference-School, Minsk 2009, pp. 103--107,ArXiv: 0807. 3485 .

[52] Zel'dovich,Y.B., 1968, The Cosmological Constant and Elementary Particle Theory, Sov.Phys.Uspehi, 11, 381-393.

[53] Weinberg, S., 1989, The Cosmological Constant Problem. Rev. Mod. Phys., 61, 1-23.

[54] Perlmutter, S. et al.,1999, Measurements of Omega and Lambda from 42 high redshift supernovae., Astrophys. J ,517, 565-586; Riess A. G. et al.,1998, Observational evidence from supernovae for an accelerating universe and a cosmological constant., Astron. J. ,116, 1009-1038; Riess A. G. et al., 1999, BV RI light curves for 22 type Ia supernovae. Astron. J ,117, 707-724.

[55] Hooft, G. 'T.,1993, Dimensional reduction in quantum gravity.Essay dedicated to Abdus Salam gr-qc/9310026, 15pp.

[56] Hooft, G. 'T.,2000, The Holographic Principle, hep-th/ 0003004,15pp.; L.Susskind, 1995, The World as a hologram. J. Math. Phys, 36, 6377-6396.

[57] Bousso, R. The Holographic principle, 2002, Rev. Mod. Phys, 74, 825-874.

[58] Bousso, R. A, 1999, Covariant entropy conjecture. JHEP ,007 , 004--021

[59] Cohen,A., Kaplan, D., Nelson, A.,1999, Effective field theory, black holes, and the cosmological constant., Phys. Rev. Lett ,82, 4971-4974.

[60] Balazs,C., and Szapudi,I.,2006, Naturalness of the vacuum energy in holographic theories., hep-th/0603133, 4pp.

[61] Fischler,W., and Susskind, L.,1998, Holography and cosmology. hep-th/9806039, 7pp.

[62] Shalyt-Margolin, A.E., and Strazhev, V. I.,2007, Dark Energy and Deformed Quantum Theory in Physics of the Early Universe. In Non-Eucleden Geometry in Modern Physics. Proc. 5-th Intern. Conference of Bolyai-Gauss- Lobachevsky (BGL-5), edited by $\mathrm{Yu}$. Kurochkin and $\mathrm{V}$. Red'kov,Minsk, 2007, 173-178.

[63] Shalyt-Margolin, A.E., and Strazhev, V. I. ,2009, Vacuum Energy and Small Parameter at Planck Scale, Nonlin. Phen. in Compl. Syst., 12,102-105.
[64] Balesku, R.,1975 Equilibruim and Nonequilibruim Statistical Mechanics,v.1,A Wiley Interscience Publications, New York-London-Sydney-Toronto.

[65] Bazarov, I.P.,1991, Thermodynamics, Moskow, Press "Higher School".

[66] Gyarmati, I., 1974, Non-Equilibruim Thermodynamics. Field Theory and Varitional Principles, Springer-Verlag, Berlin-Heidelberg-New York.

[67] Tao Zhu, Ji-Rong Ren, Ming-Fan Li.,2009, Influence of Generalized and Extended Uncertainty Principle on Thermodynamics of FRW universe. Phys.Lett.B.,674, 204-209.

[68] J.A.Wheeler,1957, On the nature of quantum geometrody-namics. Ann. Phys., 2, 604--632; J.A. Wheeler,1962, Geometrodynamics (Academic Press, London,1962); J.A. Wheeler,1964, Geometrodynamics and the issue of the final state, in Relativity, Groups and Topology, eds. B.S. and C.M. DeWitt (Gordon and Breach, New York, 1964); J.A. Wheeler,1964, Super- space and the nature of quantum geometrodynamics. in Battelle Rencontres: 1967,Lectures on Mathematics and Physics, eds. C. DeWitt and J.A. Wheeler (W. Benjamin and Co., New York, 1968).

[69] C.W. Misner, K.S. Thorne, and J.A. Wheeler, 1973, Gravitation, (Freeman, San Francisco, 1973).

[70] C.J. Isham,1997, Structural issues in quantum gravity. in Proceedings of the 14th International Conference on General Relativity and Gravitation (World Scientific, Singapore, 1997).

[71] Garay,L.,1999, Quantum evolution in spacetime foam. Int.J.Mod.Phys.A, 14, 4079-4120.

[72] Verlinde, E., 2011, On the Origin of Gravity and the Laws of Newton, JHEP,1104,029, arXiv:1001.0785.

[73] Padmanabhan,T.,2010, Equipartition of energy in the horizon degrees of freedom and the emergence of gravity. Mod. Phys. Letts. A, 25, 1129-1136, arXiv:0912.3165.

[74] Turyshev, S.G.,2010,Experimental tests of general relativity: recent progress and future directions. Phys. Usp., 52, 1-34

[75] Dvali,G.,2004, Infrared Modification of Gravity. arXiv:hep-th/0402130

[76] Patil, S.P., 2009,Degravitation, Inflation and the Cosmological Constant as an Afterglow. JCAP,0901, 017--035.

[77] Rubakov, V. A., and Tinyakov,P. G., 2008, Infrared- modified gravities and massive gravitons. Phys.Usp, 123., 759-792; Nikiforova,V., Randjbar-Daemi, S., Rubakov V., 2009, Infrared Modified Gravity with Dynamical Torsion. Phys. Rev.D, 80, 124050. 